\title{
Targeting of FK506 binding protein 5 by miR-203 affects the progression of breast cancer via regulating the fatty acid degradation pathway and potential drug-repurposing
}

\author{
DAN YANG $^{1}$, YAQIN FAN $^{2}$, BEIBEI XIE $^{1}$ and $\mathrm{JIE} \mathrm{YANG}^{2}$ \\ ${ }^{1}$ Faculty of Health, Yantai Nanshan University, Yantai, Shandong 265713; ${ }^{2}$ Department of Obstetrics \\ and Gynecology, Yuncheng County People's Hospital, Heze, Shandong 274700, P.R. China
}

Received November 23, 2020; Accepted February 5, 2021

DOI: $10.3892 / \mathrm{ol} .2021 .12607$

\begin{abstract}
Increasing number of studies have suggested that microRNA (miR)-203 is a potential prognostic marker for breast cancer. However, the specific molecular mechanism underlying the effects of miR-203 remains unknown. The present study aimed to explore the molecular target and underlying mechanisms of action of miR-203 in breast cancer via bioinformatics analysis and cellular assays, such as wound healing assay and western blotting. In the present study, 17 candidate target genes of miR-203 were identified in the downregulated differentially expressed genes from Affymetrix microarray and TargetScan 7.2 database. Subsequently, FK506 binding protein 5 (FKBP5) was considered as the miR-203 target by 3 different hub gene analysis methods (EcCentricity, Betweenness and Stress). FKBP5 protein expression was significantly downregulated in SUM159 cells transfected with miR-203 mimics compared with SUM159 cells transfected with miR-203 negative control (NC) in western blot analysis. High expression of FKBP5 was associated with poor prognosis in breast cancer based on the results obtained from the Kaplan-Meier Plotter database. In addition, the wound healing assay indicated that the inhibition of migration due to miR-203 overexpression in SUM159 cells was reversed by FKBP5 overexpression. These results suggested that miR-203 may directly target FKBP5. In addition, Gene Set Enrichment Analysis and Kyoto Encyclopedia of Genes and Genomes (KEGG) pathway
\end{abstract}

Correspondence to: Dr Jie Yang, Department of Obstetrics and Gynecology, Yuncheng County People's Hospital, 184 Tangta Road, Heze, Shandong 274700, P.R. China

E-mail: yangjie20200101@qq.com

Abbreviations: KEGG, Kyoto Encyclopedia of Genes and Genomes; GSEA, Gene Set Enrichment Analysis; DEGs, differentially expressed genes; PPI, protein-protein interaction network; Cntrl, control; miRNA/miR, microRNA

Key words: miR-203, breast cancer, target genes, KEGG, virtual screening enrichment analysis revealed that miR-203 might play a role in breast cancer via the 'fatty acid degradation' KEGG pathway. Notably, the levels of fatty acids were significantly reduced in SUM159 cells transfected with miR-203 mimics compared with SUM159 cells transfected with miR-203 NC when assessed by the fatty acid content assay. Finally, virtual screening analysis revealed that ZINC000003944422 may be a potential inhibitor of FKBP5. In summary, the present study demonstrated that miR-203 may directly target FKBP5 in breast cancer via fatty acid degradation and potential drugs, hence providing a novel treatment approach for breast cancer.

\section{Introduction}

Breast cancer is one of the most widespread types of cancer among women worldwide and the most common cause of cancer-related deaths (11.6 1\% of the total cancer deaths) according to GLOBCAN $2018(1,2)$. It was estimated that in $2019,>300,000$ new cases of breast cancer would be diagnosed in the USA, and $\sim 41,760$ women will die from this disease (3). Hence, identification of novel prognostic markers and therapeutic targets are urgently needed for breast cancer. It has been reported that microRNA (miRNA/miR)-203 is abnormally expressed in several sub-types of breast cancer, such as circulating tumor cells positive metastatic breast cancer and triple-negative breast cancer suggesting that miR-203 may be used as potential prognostic marker $(4,5)$. In addition, a study demonstrated that miR-203 could attenuate breast cancer cell viability and migration ability in vitro via reducing the abundance of Runt-related transcription factor 2 in MDA-MB-231 cells (6).

The role of miR-203 in cancer has been investigated in some studies (7-9). For example, miR-203 induced mesenchymal to epithelial transition via repressing Snail family transcriptional repressor 2 in prostate cells (7). In addition, miR-203 affected the proliferation of ovarian cancer cells via the JAK-STAT pathway and regulated the proliferation and apoptosis of ovarian cancer cells by targeting the inhibition of SOCS3 expression (8). miR-203 also suppressed bladder cancer cell growth by targeting Twist family bHLH transcription factor 1 (9). However, the mechanism of action of miR-203 and target genes in breast cancer remain elusive. 
Hence, identifying the targets and key pathways of miR-203 in breast cancer is of great importance.

High-throughput transcriptome technology provides insight into the expression profile of hundreds to tens of thousands of genes (10). In addition, pathway analysis technologies allow the mapping of gene expression data as pathway maps based on their respective functional annotation and known molecular interactions (11). Hence, the current study aimed to investigate the mechanism and target genes of miR-203 in breast cancer by combining bioinformatics analysis and in vitro experiments, which may help to improve the treatment and outcome of patients with breast cancer. The present study aimed to identify potential drugs by conducting a virtual screening analysis for miR-203 targets, which may provide a faster and cheaper strategy for expanding the arsenal of approved breast cancer drugs.

\section{Materials and methods}

Cell culture and miRNA transfection. The cell line SUM159 breast cancer was obtained from the Chinese Academy of Sciences Cell Bank, and cells were propagated in a $51 \% \mathrm{CO}_{2}$ atmosphere at $37^{\circ} \mathrm{C}$ and maintained in Ham's F-12 Nutrient Mixture (F12; cat. no. 21700075; Thermo Fisher Scientific, Inc.) with $101 \%$ (vol/vol) fetal bovine serum (FBS; cat. no. 10270-106; Gibco; Thermo Fisher Scientific, Inc.) and $11 \%$ (vol/vol) penicillin/streptomycin (cat. no. 10378016, Thermo Fisher Scientific, Inc.). The miR-203 mimics and scrambled controls (miR-203 NC) were synthesized by Shanghai GenePharma Co., Ltd. The sequences were: miR-203 mimics, 5'-TGCTTTGGCCACTGACTGTCC-3'; miR-203 negative control (NC), 5'TCGCCACATGATCGCCTAAGT-3'. Cells were seeded into 6-well plates at a density of $1 \times 10^{5}$ cells/well and cultured in fresh F12 medium 101\% FBS and without antibiotics $24 \mathrm{~h}$ prior to transfection. Subsequently, cells were transfected with miR-203 mimics or miR-203 NC using Lipofectamine ${ }^{\circledR} 3000$ (Invitrogen; Thermo Fisher Scientific, Inc.) at a final concentration of $100 \mathrm{nmol} / \mathrm{l}$ in each group, according to the manufacturer's instructions. Transfected cells were cultured at $37^{\circ} \mathrm{C}$ for $6 \mathrm{~h}$ prior to the replacement of complete medium. pCMV6-XL5-FKBP5 vector for the overexpression of FKBP5 and empty control vector pCMV6-XL5 were purchased from OriGene Technologies Inc. (cat. no. SC117569, PCMV6XL5). Cells were separately transfected with miR-203 mimics (miR-203), pCMV6-XL5-FKBP5 and miR-203 mimics (FKBP5) or Cntrl (empty control vector pCMV6-XL5 and miR-203 NC) using Lipofectamine ${ }^{\circledR} 3000$ (Invitrogen; Thermo Fisher Scientific, Inc.), according to the manufacturer's instructions. Transfected cells were cultured at $37^{\circ} \mathrm{C}$ for $6 \mathrm{~h}$ prior to the replacement of complete medium. Cells were harvested for subsequent experiments $24 \mathrm{~h}$ following transfection and all experiments were performed in triplicate.

Reverse transcription-quantitative (RT-q) PCR. Total RNA from SUM159 cells transfected with miR-203 mimics or miR-203 NC was extracted using TRIzol ${ }^{\circledR}$ reagent (Invitrogen; Thermo Fisher Scientific Inc.) and cDNA was synthesized using iScript ${ }^{\mathrm{TM}}$ cDNA Synthesis Kit (Biorad Laboratories Inc.) following the manufacturer's instructions. $\mathrm{iQ}^{\mathrm{TM}} \mathrm{SYBR}$
Green supermix (Biorad Laboratories Inc.) was used to perform RT-qPCR. A 7500 HT Fast Real-Time PCR system (Applied Biosystems; Thermo Fisher Scientific Inc.) was used to cycle and quantify reactions. The thermocycling conditions were as follows: denaturation at $95^{\circ} \mathrm{C}$ for $20 \mathrm{sec}$, followed by 40 cycles of $95^{\circ} \mathrm{C}$ for $10 \mathrm{sec}, 60^{\circ} \mathrm{C}$ for $20 \mathrm{sec}$ and $70^{\circ} \mathrm{C}$ for $10 \mathrm{sec}$. Relative gene expression levels of miR-203 were evaluated. U6 was used as the miRNA endogenous normalization control. The relative expression levels of FKBP5 were evaluated relative to glyceraldehyde 3-phosphate dehydrogenase (GAPDH). The primer sequences used were as follows: miR-203 forward 5'-GGGGTGAAATGTTTAGGAC-3; reverse 5'-CAGTGCGTGTCGTGGAGT-3'; U6 forward 5'-CTCGCTTCGGCAGCACA-3', reverse 5'-AACGCTTCA CGAATTTGCGT-3'; FKBP5 forward 5'-GGGGTGAAATG TTTAGGAC-3; reverse 5'-CAGTGCGTGT CGTGGAGT-3'; and GAPDH forward 5'-TCAAGAAGG TGGTGAAGCAG-3', reverse 5'-CGCTGTTGAAGTCAGAGGAG-3'. The relative expression of each target amplicon was calculated using the $2^{\Delta \Delta \mathrm{Cq}}$ method (12).

Data preprocessing and analysis of differentially expressed genes (DEGs). To investigate the downstream molecular pathways of miR-203, potentially mediating its effects in breast cancer, gene expression arrays were carried out in SUM159 cells transfected with miR-203 mimics or miR-203 NC. The changes in the miRNA expression levels were detected in total RNA isolated by a TRIzol ${ }^{\circledR}$ reagent (Invitrogen; Thermo Fisher Scientific Inc.). Reverse transcription and hybridization were performed using the Human Genome U133 Plus 2.0 Array (cat. no. 902482; Affymetrix, Inc.). This microarray had 9,921 probe sets representing $\sim 6,500$ genes and interrogated $>47,000$ transcripts. The raw data was submitted to National Microbiology Data Center (SUB1610765979725, http://nmdc. $\mathrm{cn} /$ resource/attachment/ detail/NMDCX0000106). The original expression profiles were transformed into a matrix using Affy package in R v. 3.6.1 (13). Subsequently, the Limma package v. 3.40.2 (14) was utilized to identify DEGs. The threshold was set at $\mathrm{P}<0.05$ and $\mid \log ($ fold change) $\mid>1$. A volcano plot and heatmap of DEGs were constructed using the pheatmap v.1.0.12 package in R v. 3.6.1 (https://cran.r-project. org/web /packages/pheatmap/index.html).

Pathway enrichment analysis. Kyoto Encyclopedia of Genes and Genomes (KEGG, https://www.genome.jp/kegg/) is a database used for the systematic analysis of gene function via linking genomic information with high-level systemic function (15). After obtaining the DEGs, the ClusterProfiler 3.12.0 package (16) was utilized to perform KEGG enrichment analyses, with a cut-off value of $\mathrm{P}<0.05$. Simultaneously, Gene Set Enrichment Analysis (GSEA) based on KEGG gene sets in the MSigDB database (17) was carried out to identify the enriched pathways between SUM159 cells transfected with or without miR-203, with cut-off values of false discovery rate $(\mathrm{FDR})<251 \%$ and $\mathrm{P}<0.05$, which were recommended by GSEA v.4.0.3 (17).

Predicting the targets of miR-203, protein-protein interaction network (PPI) and core genes in the PPI network. The potential targets of miRNA-203 were predicted using 
TargetScan 7.2 (18). Subsequently, the intersection of genes identified by Targetscan 7.2 database and downregulated DEGs were then regarded as candidate targets of miR-203. Additionally, the human PPI network was retrieved from the Search Tool for the Retrieval of Interacting Genes (STRING) database (19) and constructed using both TargetScan and downregulated DEGs. Hub genes are considered important biomarkers (10); therefore, the EcCentricity, Betweenness and Stress algorithms were utilized to identify the hub genes by cytoHubba plugin of Cytoscape v.3.7.1 (20). In addition, the prognostic value of the hub genes was validated with the Kaplan Meier plotter (KMplot) database (21).

Validation of transcriptome analyses by western blot analysis. SUM159 cells transfected with miR-203 mimics or miR-203 $\mathrm{NC}$ were lysed at $4^{\circ} \mathrm{C}$ in buffer $(1 \mathrm{mM} \beta$-glycerophosphate, $2.5 \mathrm{mM}$ sodium pyrophosphate, $20 \mathrm{mM}$ Tris- $\mathrm{HCl}$ (pH 7.4), $1 \%$ Triton $\mathrm{X}-100,150 \mathrm{mM} \mathrm{NaCl}, 1 \mathrm{mM}$ EGTA, $1 \mathrm{mM}$ EDTA and $1 \mathrm{mM} \mathrm{Na} \mathrm{VO}_{4}$ ) supplemented with protease inhibitor cocktail (1:1,000; cat. no. P8340; Merck KGaA). The protein concentration was determined with a bicinchoninic acid (BCA) protein assay kit (cat. no. 23225; Thermo Fisher Scientific Inc.). Protein samples $(20 \mu \mathrm{g})$ were separated by $121 \%$ SDS-PAGE and then transferred onto a PVDF membrane. Following blocking with $51 \%(\mathrm{w} / \mathrm{v})$ skimmed milk in wash buffer (TBS and $0.051 \%$ Tween-20) for $1 \mathrm{~h}$ at room temperature, the membranes were incubated $4^{\circ} \mathrm{C}$ with the following primary antibodies: anti-GAPDH $(1: 10,000$; cat.no. G9545; Sigma-Aldrich; Merck KGaA) and anti-FK506 binding protein 5 (FKBP5; 1:1,000; cat. no. sc-271547; Santa Cruz Biotechnology, Inc.). Subsequently, the membranes were incubated with a corresponding HRP-conjugated IgG secondary antibody (1:5,000; cat. nos. ab6721 and ab6789; Abcam.) at room temperature for $1 \mathrm{~h}$. The immunoreactive signals were scanned and quantified using the ImageQuant LAS 4000 software v.1.2 (Cytiva).

Wound healing motility assay. Cells were harvested for scratch assay at $24 \mathrm{~h}$ following transfection and all experiments were performed in triplicate. Briefly, SUM159 cells were seeded into a 12 -well plate at a density of $1 \times 10^{5}$ cells/well. The wound was generated when cells reached $90-951 \%$ confluency by scratching the surface of the plates with a pipette tip. Subsequently, the cells were washed thrice with PBS to remove cell debris and incubated for $15 \mathrm{~h}$ with fresh F12 medium $11 \%$ FBS and without antibiotics. Finally, the migrated cells were imaged under a light phase-contrast microscope (magnification, $\mathrm{x} 40$ ). The migration distance in each group was measured using ImageJ v.1.8.0 (National Institutes of Health) (22) and statistically analyzed.

Identifying potential drug repurposing candidates. FKBP5 3D structure was downloaded from the Protein Data Bank (PDB; 6SAF; https://www.rcsb.org/) and its biding sites were identified by Maestro 2019-1 software (Schrödinger, LLC) (23). Subsequently, a library of 2,106 Food and Drug Administration (FDA)-approved drugs obtained from the ZINC15 database (24) was constructed. Finally, virtual screening and molecular docking was performed using the Maestro 2019-1 (Schrödinger, LLC) software to identify potential drug-repurposing candidates. The best potential drug was determined based on the glide score (25).

Free fatty acid content assay. SUM159 cells were transfected with miR-203 or miR-203 NC as described earlier. Then, SUM159 cells were seeded in 6-well plates at a density of $1 \times 10^{5}$ cells/well with complete medium. Subsequently cells were harvested for fatty acid content assay after $24 \mathrm{~h}$. Fatty acids were extracted with a Free Fatty Acid Content assay kit (cat. no. D799794, Sangon Biotech, Co., Ltd.) according to the manufacturer's instructions. As the manufacturer's protocol, copper ions could combine free fatty acid to form fatty acid copper salt. Its content is in direct proportion to free fatty acids. The content of fatty acid can be calculated by measuring the content of copper ions with copper reagent by colorimetry.

Statistical analysis. All experiments were repeated at least 3 times. Quantitative data are expressed as the mean \pm standard error of the mean (SEM). All statistical analyses were carried out using the SPSS 18.0 software (SPSS, Inc.). The figures were generated using tGraph Prism 7.0 software (GraphPad Software, Inc.). All data were checked for normal distribution and homogeneity of variance using Kolmogorov-Smirnov one-sample and Levene's tests. Statistical evaluation of the data was performed by using the unpaired Student's t-test or Kruskal Wallis non-parametric test with a post hoc Dunn's test. Survival analysis was performed using KMplot with an auto-selected best cutoff and log-rank test. $\mathrm{P}<0.05$ was considered to indicate a statistically significant difference.

\section{Results}

Identification of DEGs. To assess the transfection efficiency of miR-203 in SUM159 cells, RT-qPCR was performed and the results demonstrated that the expression of miR-203 was significantly increased by 2.6-fold compared with the control group (SUM159 cells transfected with miR-203 NC) $(\mathrm{P}<0.05 ;$ Fig. S1). The box plots for the expression values for all genes in each sample following normalization by $\mathrm{R}$ package Limma 3.40.2 are represented in Fig. 1A. On comparing miR-203-overexpressing SUM159 cells with Cntrl (SUM159 cells transfected with miR-203 NC) counterparts, 1,101 DEGs were identified with a threshold of $\mathrm{P}<0.05$ and $\mid \operatorname{logFCl}>1$ (Table SI). In addition, a heatmap of DEGs was constructed to visualize their expression levels in different samples (Fig. 1B). Volcano plots of DEGs drawn by pheatmap 1.0.12 for the two treatment groups were presented in Fig. 1C. The significantly downregulated genes are indicated by green dots, and the significantly upregulated ones by red dots. Black dots indicate genes with no significant differences in gene expression.

Identification of key pathways between KEGG and GSEA. To further investigate the function of the aforementioned DEGs, KEGG pathway enrichment analysis was carried out. Pathways which were significantly enriched $(\mathrm{P}<0.05)$ were identified using the ClusterProfiler package. Based on the KEGG database, DEGs were significantly enriched in the 'tryptophan metabolism', 'fatty acid degradation' and 


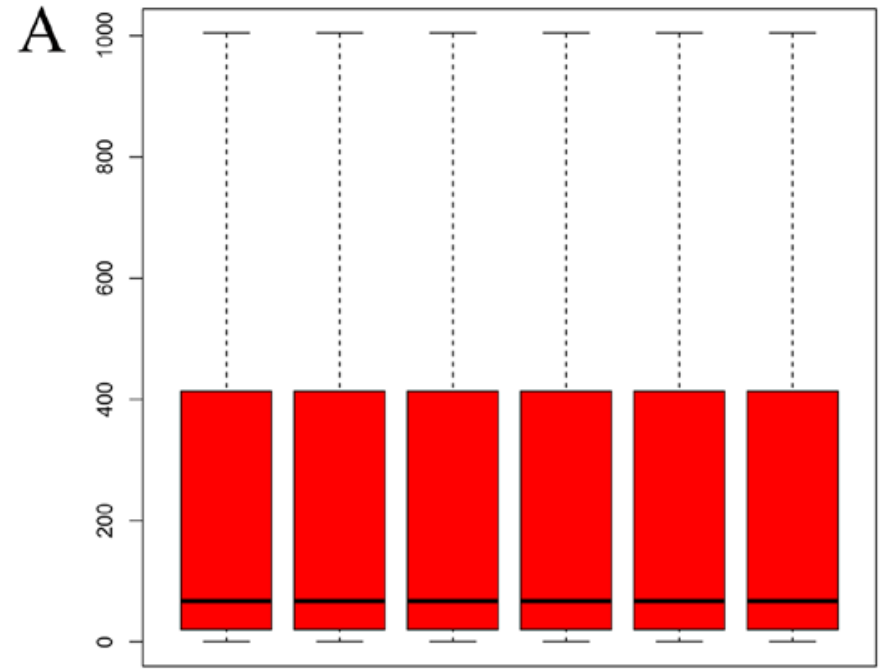

Control-1 Control-2 Control-3 miR-203-1 miR-203-2 miR-203-3 Volcano

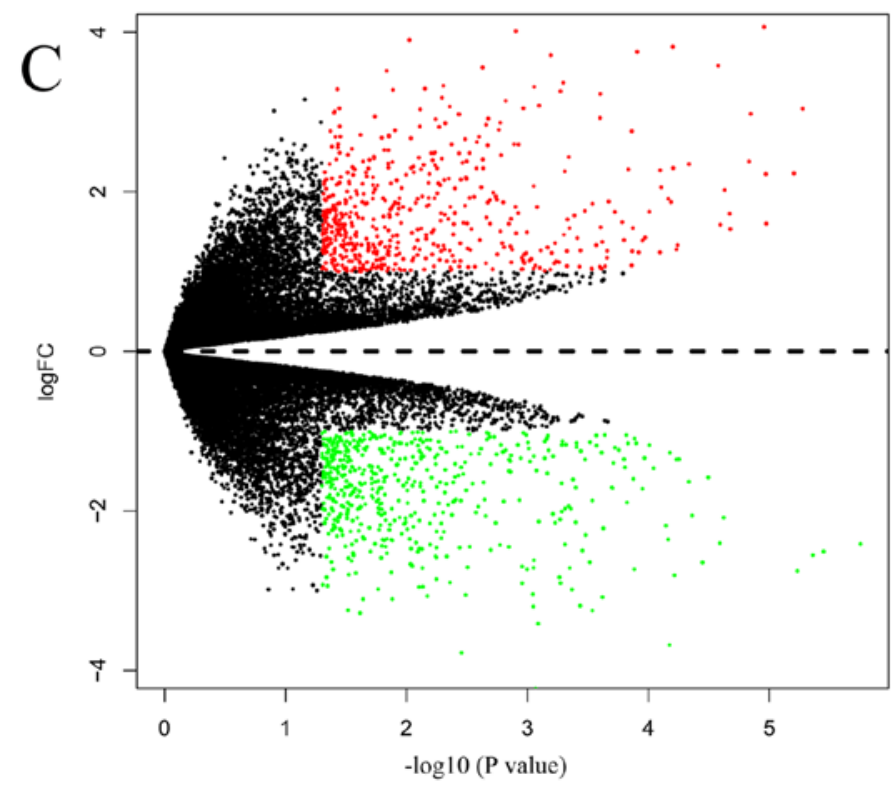

B

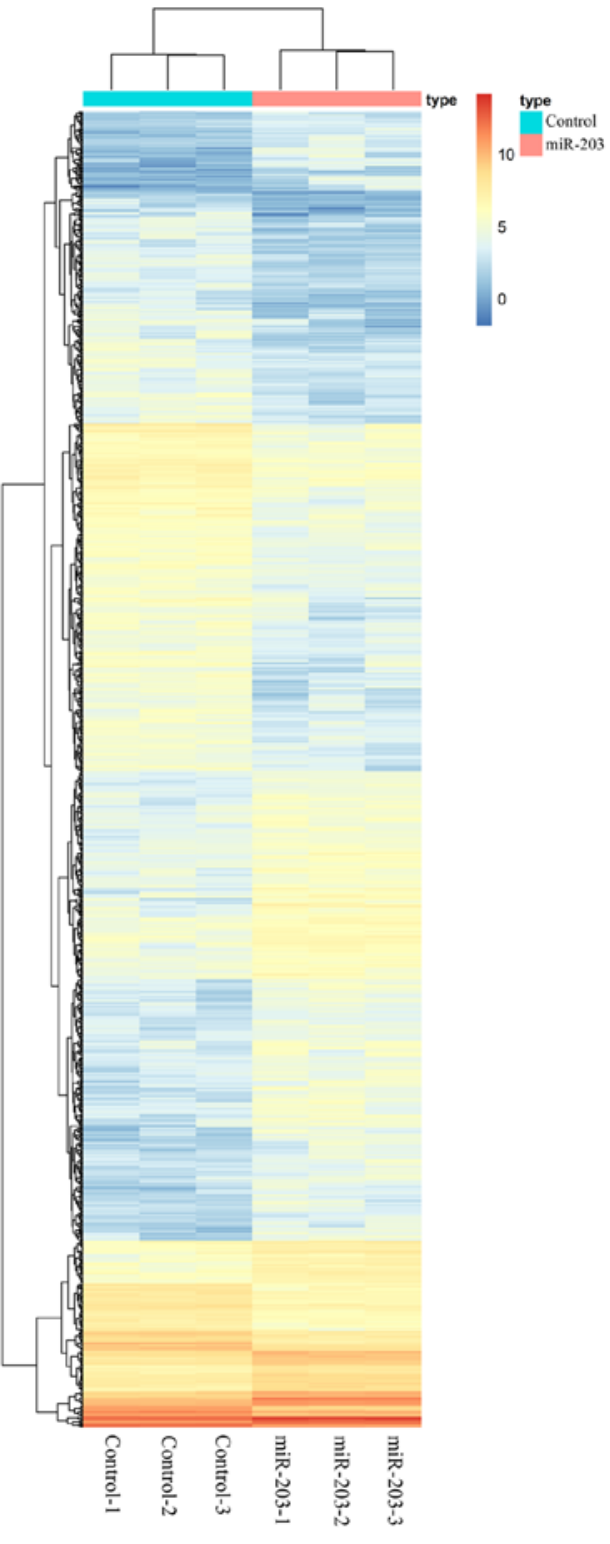

Figure 1. Screening of differential mRNA expression between miR-203 mimic and miR-203 NC groups by Limma v.3.40.2 (A) The black lines in the boxes indicate the median value of each dataset, determining the degree of standardization of data through their distribution. Following normalization by Limma v3.40.2, the black lines in the box are almost in the same straight line indicating a good degree of standardization. (B) Heat map of DEGs between SUM159 cells transfected with miR-203 mimics and miR-203 NC by pheatmap v.1.0.12. (C) Volcano plot of all unique genes obtained from the differential mRNA expression analysis. The significantly downregulated genes are indicated by green dots, and the significantly upregulated ones by red dots. Black dots indicate genes with no significant differences in gene expression. Control, SUM159 transfected with miR-203 NC; miR-203, SUM159 transfected with miR-203 mimics; DEGs, differentially expressed genes; miR, microRNA; NC, negative control.

'primary bile acid biosynthesis' pathways (Fig. 2A). GSEA was carried out to summarize genome-wide gene expression changes into gene sets, other than DEGs, between Cntrl (SUM159 cells transfected with miR-203 NC) and treatment groups (SUM159 cells transfected with miR-203). A total of 25 pathways enriched in the $\mathrm{Cntrl}$ group $(\mathrm{P}<0.05$ and FDR $<25 \%$, Table SII), and 24 in the treatment group were predicted by applying GSEA $(\mathrm{P}<0.05$ and $\mathrm{FDR}<25 \%$; Table SIII). Between KEGG pathway enrichment analysis and GSEA, one overlapping pathway was obtained, namely the 'fatty acid degradation' pathway (Fig. 2A and B). Hence, the fatty acid content was evaluated using the free fatty acid content assay kit and the results demonstrated that the levels of fatty acids were significantly reduced in the miR-203 group compared with the control group (SUM159 cells transfected with miR-203, P<0.05; Fig. S2).

Predicted target genes of miR-203, PPI network, and core genes in the PPI network. A total of 1481 target genes of miR-203 were predicted using the Targetscan database. Among them, 17 genes were downregulated DEGs (Fig. 3A). Subsequently, to reveal the interactions among target genes, the genes were subjected to screening in the STRING database. Only interactions with a combined score of $>0.15$ were considered significant (19). The PPI network was comprised of 17 nodes (8 nodes with combined score $>0.15)$ and 12 edges (Fig. 3B). FKBP5 was identified as the top overlapping gene among 3 ranking methods (EcCentricity, Betweenness and 

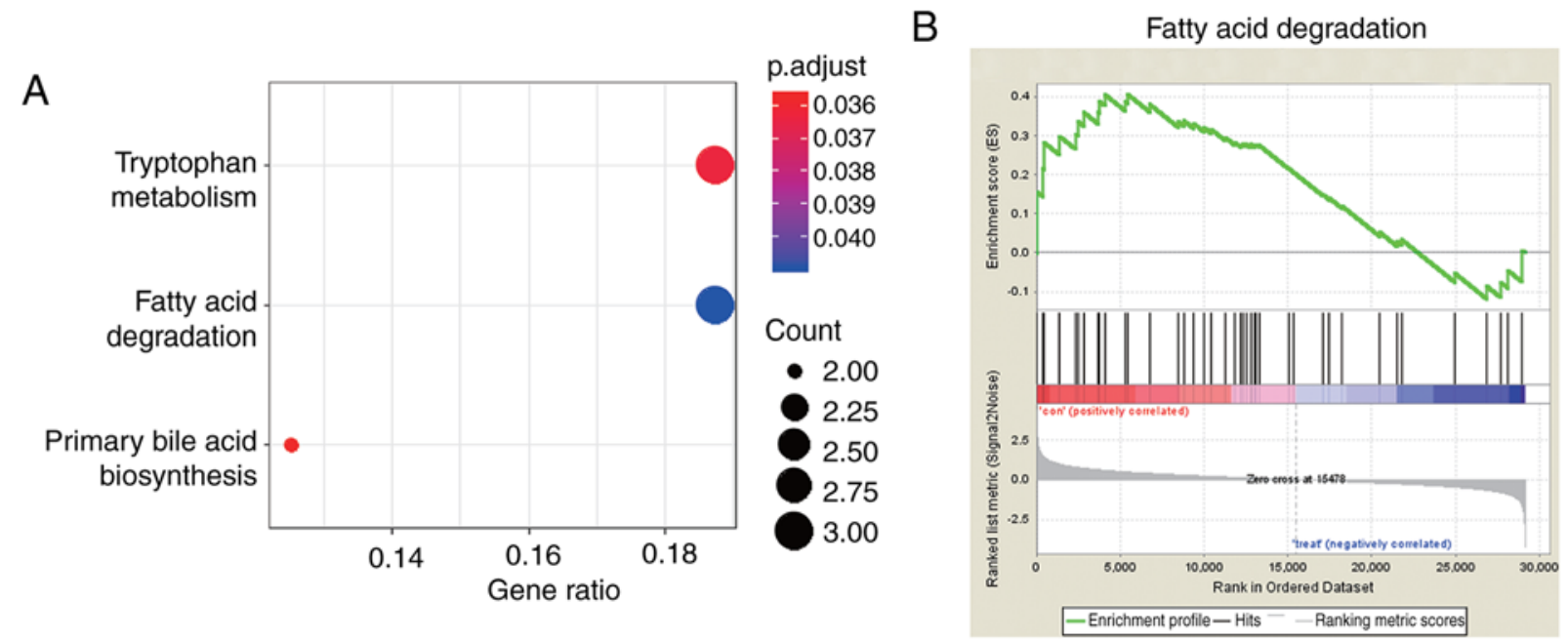

Figure 2. KEGG pathway enrichment and GSEA between SUM159 cells transfected with miR-203 mimic and miR-203 NC. (A) KEGG pathway enrichment analysis of DEGs using the ClusterProfiler package in $\mathrm{R}(\mathrm{P}<0.05)$. (B) GSEA showing the association between the miR-203 expression levels and KEGG gene sets in the MSigDB database (FDR <25\%; P<0.05). KEGG, Kyoto Encyclopedia of Genes and Genomes; GSEA, Gene Set Enrichment Analysis; DEGs, differentially expressed genes; FDR, false discovery rate.

A

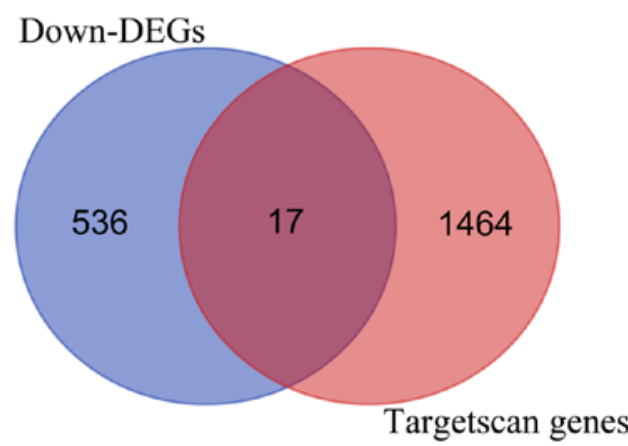

$\mathrm{C}$

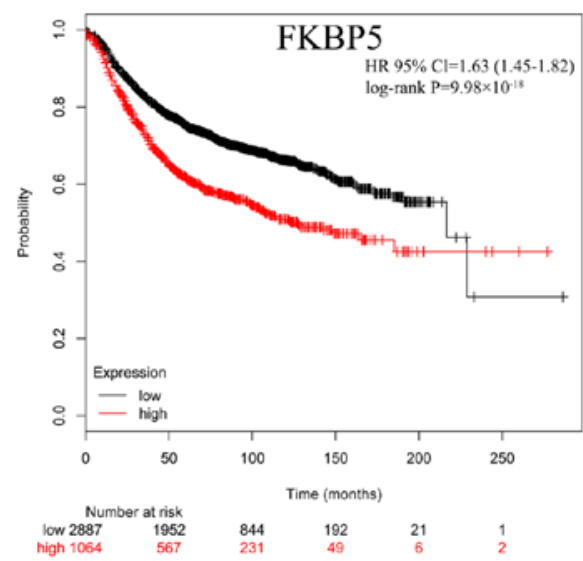

B

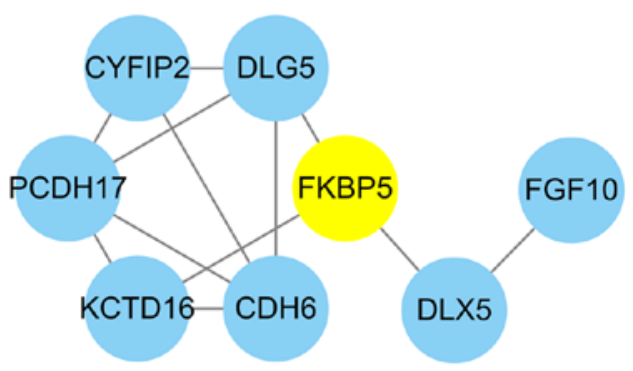

$\mathrm{D}$

\section{Cntrl miR-203}
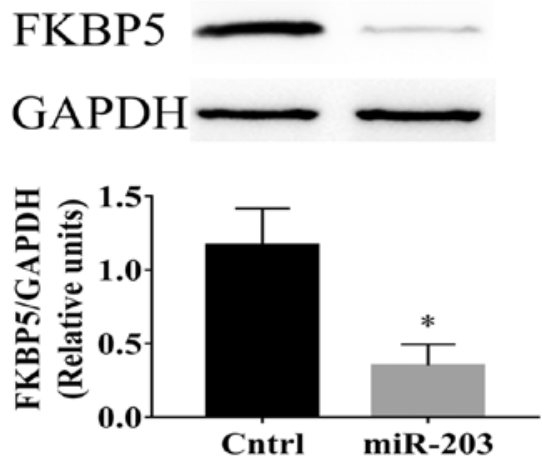

Figure 3. Prediction of miR-203 targets, PPI network, and core genes in the PPI network. (A) A total of 17 target genes were identified between Down-DEGs and Targetscan 7.2. (B) PPI network of target genes. (C) Kaplan-Meier plot was used to estimate the overall survival of patients with breast cancer based on the expression of FKBP5. (D) The expression levels of FKBP5 in SUM159 cells transfected with miR-203 mimics or miR-203 NC were determined by western blot analysis. Data are expressed as the mean $\pm \mathrm{SD}(\mathrm{n}=3) .{ }^{*} \mathrm{P}<0.05$ compared with the Cntrl group (SUM159 cells transfected with miR-203 NC; unpaired t-test). PPI, protein-protein interaction; Down-DEGs, downregulated differentially expressed genes; FKBP5, FK506 binding protein 5; Cntrl, control; HR, hazard ratio; miR, microRNA

Stress), using the cytoHubba plugin (Table SIV). In addition, overall survival analysis based on the Kmplot database with an auto-selected best cutoff and log-rank test was performed to further verify the role of the FKBP5 hub gene. The analysis revealed that FKBP5 was significantly associated with breast cancer (log-rank $\mathrm{P}<0.05$ and HR=1.63; Fig. 3C). This finding was further verified by detecting the expression levels of FKBP5 using western blot analysis. Compared with the Cntrl group (SUM159 cells transfected with miR-NC), the protein expression levels of FKBP5 were significantly downregulated in the 

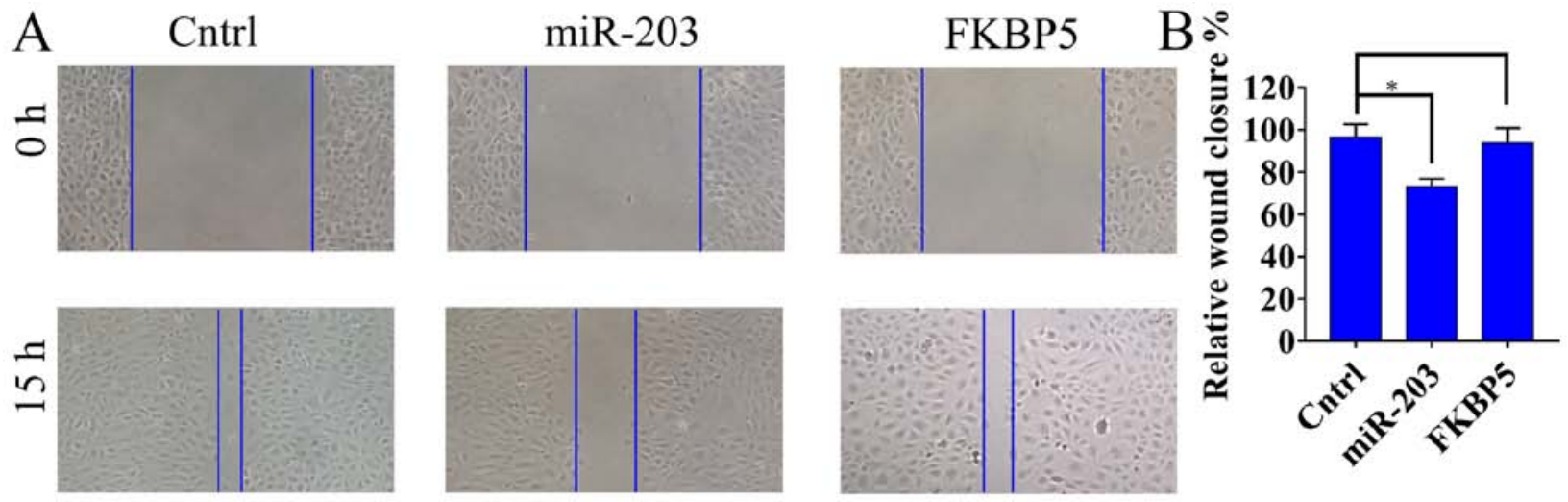

C

pCMV6-XL5: 4479 bp

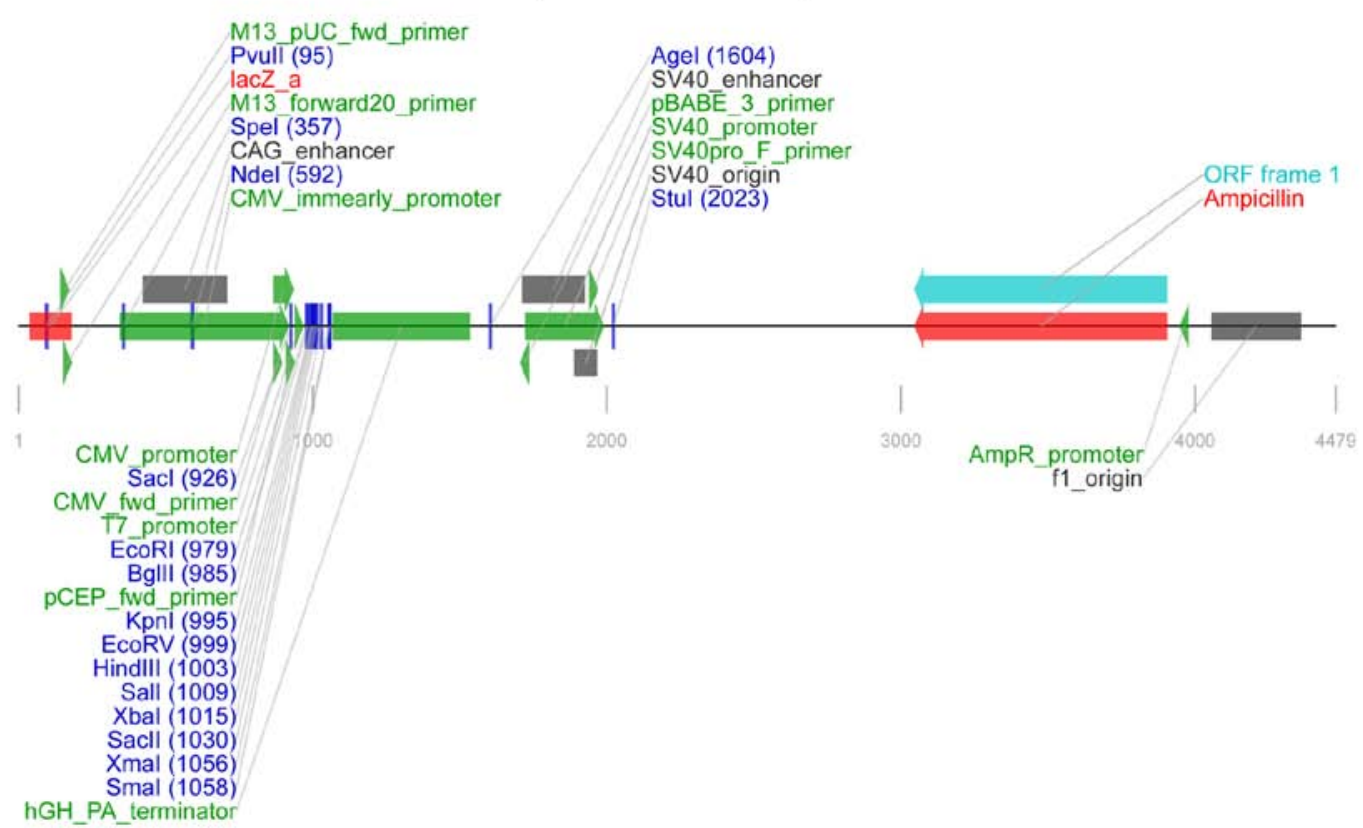

Figure 4. Migration capability of SUM159 breast cancer cells. To evaluate the migration ability of Cntrl (SUM159 cells transfected with miR-203 NC and empty control vector pCMV6-XL5), miR-203 (SUM159 cells transfected with miR-203 mimic), and FKBP5 (SUM159 cells transfected with pCMV6-XL5-FKBP5 vector), wound healing assays were carried out under a light phase-contrast microscope (magnification, $\mathrm{x} 40$ ) (A) Representative images showing the wound healing at 0 and $15 \mathrm{~h}$. Images were captured using an inverted microscope and the wound area was calculated at the indicated time points using Image J v.1.8.0 (B) Graph represents the normalized (to $\mathrm{t}=0 \mathrm{~h}$ ) percentage of wound healing at 0 and $15 \mathrm{~h}$ following scratching. Data are expressed as the mean $\pm \mathrm{SD}$ from 6 wound-healing assays for each group. Statistical analysis was performed using Kruskal Wallis non-parametric ANOVA with Dunn's post hoc test comparing every treatment and with its corresponding control ("P<0.05). (C) The liner map of vector pCMV6-XL5. Cntrl, control; FKBP5, FK506 binding protein 5; miR, microRNA.

miR-203 group ( $\mathrm{P}<0.05$; Fig. 3D). The western blotting results were in line with those observed in DEGs.

FKBP5 upregulation restores the migration ability of miR-203 overexpressing SUM159 cells. To assess the transfection efficiency of miR-203 and FKBP5 in SUM159 cells, RT-qPCR was performed. RT-qPCR results demonstrated that miR-203 and FKBP5 expression significantly increased by 2.1 -fold and 1.8-fold compared with the Cntrl group (SUM159 cells transfected with miR-NC and empty control vector pCMV6-XL5), respectively ( $\mathrm{P}<0.05$; Fig S3). In addition, to further verify that miR-203 could directly target FKBP5, scratch wound healing assays were performed in SUM159 (Cntrl, SUM159 cells transfected with miR-NC and empty control vector pCMV6-XL5), SUM159 miR-203 overexpressing (SUM159 cells transfected with miR-203), and SUM159 FKBP5 overexpressing (FKBP5, SUM159 cells transfected with miR-203 and pCMV6-XL5-FKBP5) breast cancer cells. As demonstrated in Fig. 4A, after $15 \mathrm{~h}$ the wound closure was almost the same between the Cntrl and FKBP5 groups. However, in the miR-203 group the SUM159 cell migration was slower compared with the Cntrl group $(\mathrm{P}<0.05$; Figs. 4A and B). The liner map of empty vector pCMV6-XL5 was shown in Fig. 4C.

ZINC000003944422 is considered as a potential drug repurposing candidate of FKBP5. Virtual screening (Maestro 2019-1) was utilized to identify potential drug repurposing candidates for FKBP5. Hence, the 3D protein structure of FKBP5 was downloaded from PDB (6SAF). According to the glide scores, ZINC000003944422 was the top 1 hit obtained from the structure-based virtual screening 


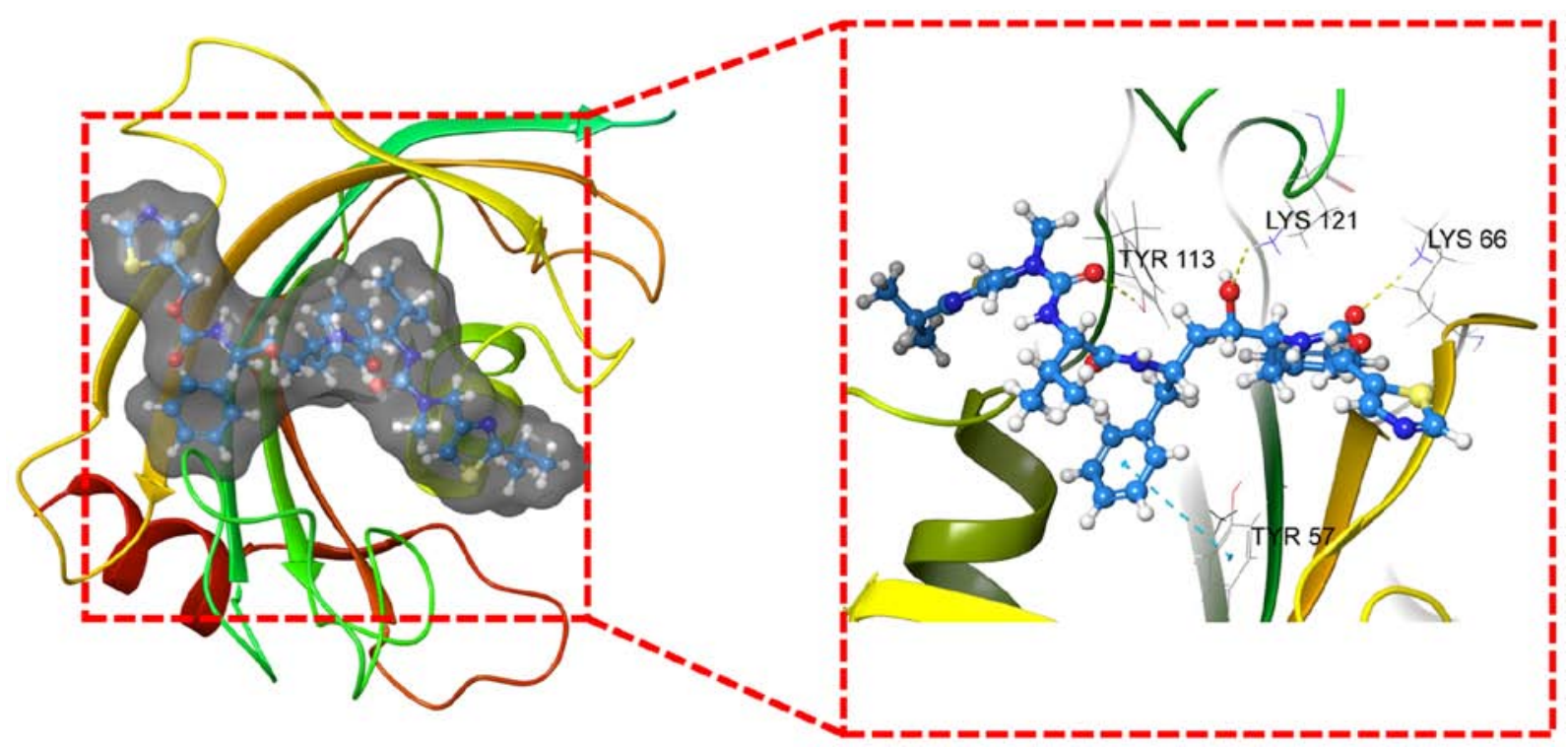

Figure 5. Potential drug-repurposing for FKBP5 by virtual screening. The 3D structure of ZINC000003944422 binding in FKBP5 is shown. Yellow and green arrows indicate hydrogen bonds and Pi interactions, respectively. Salt bridges are indicated by purple lines. FKBP5, FK506 binding protein 5; TYR, tyrosine; LYS, lysine; Pi interactions, salt bridge.

process (Table SV). The 3D structure of ZINC000003944422 is presented in Fig. 5. Additionally, hydrogen bonds and Pi interactions were identified in the ligand-protein complex between FKBP5 and ZINC000003944422 (Fig. 5). In addition, ZINC000003944422 is also known as Norvir.

\section{Discussion}

In 2019, breast cancer was the most common type of cancer and the second leading cause of cancer-related death among women in the USA after lung cancer (3). Recently, several studies have elucidated the key roles of miRNAs in regulating gene expression during cancer development. For example, high expression of miR-190 suppressed breast cancer metastasis (26). miR-21 promoted breast cancer proliferation by targeting Leucine zipper transcription factor-like 1 (LZTF1) (27). Emerging evidence has suggested that miR-203 may serve an import role in cancer (7-9). However, the specific underlying molecular mechanisms of miR-203 in breast cancer remain unclear. The present study demonstrated that miR-203 can directly target FKBP5 and is involved in the 'fatty acid degradation' pathway in breast cancer. In addition, in the present study potential drugs that inhibit FKBP5 were identified using virtual screening analysis.

To explore the underlying mechanism of miR-203 in breast cancer in the present study, SUM159 were cells were transfected. According to RT-qPCR results, the transfection efficiency in the present study was like previous studies $(28,29)$. A microarray analysis based on the SUM159 cells transfected with miR-203 mimics or miR-203 NC was conducted in the present study. According to the results of microarray, the 'fatty acid degradation' pathway was the most commonly enriched pathway in the KEGG pathway enrichment and GSEA results. In the present study, GSEA was carried out to summarize genome-wide gene expression changes in gene sets other than DEGs between Cntrl and treatment groups. Fatty acid contents were significantly decreased in SUM159 cells transfected with miR-203 mimics compared with SUM159 cells transfected with miR-203 NC. Fatty acids are fundamental substrates required for energy storage, synthesis of membranes, generation of signaling molecules and formation of lipid droplets in cancer cells (30). Overactivation of fatty acid metabolism is one of the most aberrant metabolic alterations in cancer cells, which promotes cancer cell survival and helps maintain their invasive ability (31). The results of the current study were in line with a previous one, suggesting that miR-203 inhibits lung cancer cell metastasis via targeting fatty acid binding protein 4 (FABP4) (32). In addition, another study demonstrated that fatty acid degradation may serve an important role in breast cancer cell survival and proliferation via regulating oxidative stress (33). Taken together, the results of the present study were in accordance with the ones obtained from the previous aforementioned studies. Hence, we hypothesized that overexpression of miR-203 may reduce the contents of fatty acids which in turn may inhibit cancer cell growth and metastasis.

In the present study, one hub target gene, FKBP5, was identified by PPI network and target analysis. In addition, wound healing assays indicated that miR-203 overexpression induced inhibition of migration of SUM159 cells were reversed by FKBP5 overexpression. These results suggested that miR-203 may directly target FKBP5 in breast cancer. It has been reported that FKBP5 is involved in breast and ovarian cancer $(34,35)$. Another study demonstrated that FKBP5 may act as a tumor suppressor and affect cell response to chemotherapy (36). The present study further supported the findings of previous studies, indicating that FKBP5 may be directly targeted by miR-203 in breast cancer.

In the present study, the potential drug repurposing candidates were identified using virtual screening analysis based on 2,106 FDA-approved drugs. The analysis revealed that FKBP5 may be a potential therapeutic target in breast cancer. In addition, ZINC000003944422 was found as a potential target-drug for FKBP5. Screening in the ZINC15 database in the present study also demonstrated that ZINC000003944422 was Norvir. It has been reported that Norvir inhibits the proliferation of renal 
cancer (37) and bladder cancer cells (38). In line with previous studies, the findings of the present study suggested that Norvir may improve the outcome of patients with breast cancer. Hence, drug development could be accelerated to improve the outcome of patients with breast cancer. However, further experiments should be carried out to verify the results of the current study.

The present study had limitations. The results in the present study were mainly based on SUM159 cells and the potential drug was identified by virtual screening (instead of performing laboratory experiments). Further studies based on different breast cancer cell lines are needed to verify the results of the present study. In addition, well-designed laboratory experiments are required to prove that ZINC000003944422 is a potential drug for FKBP5.

Overall, in the present study 1,101 DEGs were identified between Cntrl and breast cancer tissues. In addition, KEGG pathway enrichment and GSEA revealed that miR-203 was enriched in the 'fatty acid degradation' pathway in breast cancer. In addition, a total of 17 target genes of miR-203 were identified in the downregulated DEGs and Targetscan 7.2. To further detect the hub genes among target genes, hub gene analysis was performed using the cytoHubba plugin. In addition, virtual screening analysis predicted that ZINC000003944422 may be a potential target-drug for FKBP5. Taken together, the aforementioned findings indicated that miR-203 may directly target FKBP5 in breast cancer via fatty acid degradation and potential drugs, hence providing a novel treatment approach for breast cancer.

\section{Acknowledgements}

Not applicable.

\section{Funding}

No funding was received.

\section{Availability of data and materials}

All data generated or analyzed during this study are included in this published article. Publicly available datasets were analyzed in this study. The data can be found in the ZINC15 (http://zinc15.docking.org/) and Kmplot (https://kmplot. com/analysis/) databases.

\section{Authors' contributions}

DY and JY contributed to the study design, data collection, data interpretation and manuscript preparation. BX and YF contributed to data collection, data analysis and statistical analysis. DY and JY confirm the authenticity of all the raw data. All authors read and approved the final manuscript.

\section{Ethics approval and consent to participate}

Not applicable.

\section{Patient consent for publication}

Not applicable.

\section{Competing interests}

The authors declare that they have no competing interests.

\section{References}

1. Telli ML, Gradishar WJ and Ward JH: NCCN Guidelines Updates: Breast Cancer. J Natl Compr Canc Netw 17: 552-555, 2019.

2. Bray F, Ferlay J, Soerjomataram I, Siegel RL, Torre LA and Jemal A: Global cancer statistics 2018: GLOBOCAN estimates of incidence and mortality worldwide for 36 cancers in 185 countries. CA Cancer J Clin 68: 394-424, 2018.

3. DeSantis CE, Ma J, Gaudet MM, Newman LA, Miller KD, Goding Sauer A, Jemal A and Siegel RL: Breast cancer statistics, 2019. CA Cancer J Clin 69: 438-451, 2019.

4. Madhavan D, Zucknick M, Wallwiener M, Cuk K, Modugno C, Scharpff M, Schott S, Heil J, Turchinovich A, Yang R, et al: Circulating miRNAs as surrogate markers for circulating tumor cells and prognostic markers in metastatic breast cancer. Clin Cancer Res 18: 5972-5982, 2012.

5. Wang C, Zheng X, Shen C and Shi Y: MicroRNA-203 suppresses cell proliferation and migration by targeting BIRC5 and LASP1 in human triple-negative breast cancer cells. J Exp Clin Cancer Res 31: 58, 2012.

6. Taipaleenmäki H, Browne G, Akech J, Zustin J, van Wijnen AJ, Stein JL, Hesse E, Stein GS and Lian JB: Targeting of Runx 2 by miR-135 and miR-203 Impairs Progression of Breast Cancer and Metastatic Bone Disease. Cancer Res 75: 1433-1444, 2015.

7. Qu Y, Li WC, Hellem MR, Rostad K, Popa M, McCormack E, Oyan AM, Kalland KH and Ke XS: miR-182 and miR-203 induce mesenchymal to epithelial transition and self-sufficiency of growth signals via repressing SNAI2 in prostate cells. Int J Cancer 133: 544-555, 2013.

8. Liu HP, Zhang Y, Liu ZT, Qi H, Zheng XM, Qi LH and Wang JY: miR-203 regulates proliferation and apoptosis of ovarian cancer cells by targeting SOCS3. Eur Rev Med Pharmacol Sci 23: 9286-9294, 2019.

9. Shen J, Zhang J, Xiao M, Yang J and Zhang N: miR-203 Suppresses Bladder Cancer Cell Growth and Targets Twist1. Oncol Res 26: 1155-1165, 2018.

10. Yang F, Qiu W, Li R, Hu J, Luo S, Zhang T, He X and Zheng C: Genome-wide identification of the interactions between key genes and pathways provide new insights into the toxicity of bisphenol $\mathrm{F}$ and $\mathrm{S}$ during early development in zebrafish. Chemosphere 213: 559-567, 2018.

11. Kanehisa M, Sato Y, Furumichi M, Morishima K and Tanabe M: New approach for understanding genome variations in KEGG. Nucleic Acids Res 47D: D590-D595, 2019.

12. Livak KJ and Schmittgen TD: Analysis of relative gene expression data using real-time quantitative PCR and the 2(-Delta Delta C(T)) Method. Methods 25: 402-408, 2001.

13. Gautier L, Cope L, Bolstad BM and Irizarry RA: affy - analysis of Affymetrix GeneChip data at the probe level. Bioinformatics 20: 307-315, 2004.

14. Smyth GK, Michaud J and Scott HS: Use of within-array replicate spots for assessing differential expression in microarray experiments. Bioinformatics 21: 2067-2075, 2005.

15. Kanehisa M, Goto S, Sato Y, Furumichi M and Tanabe M: KEGG for integration and interpretation of large-scale molecular data sets. Nucleic Acids Res 40D: D109-D114, 2012.

16. Yu G, Wang LG, Han Y and He QY: clusterProfiler: An $\mathrm{R}$ package for comparing biological themes among gene clusters. OMICS 16: 284-287, 2012.

17. Subramanian A, Tamayo P, Mootha VK, Mukherjee S, Ebert BL, Gillette MA, Paulovich A, Pomeroy SL, Golub TR, LanderES, et al: Gene setenrichmentanalysis: A knowledge-based approach for interpreting genome-wide expression profiles. Proc Natl Acad Sci USA 102: 15545-15550, 2005.

18. Agarwal V, Bell GW, Nam JW and Bartel DP: Predicting effective microRNA target sites in mammalian mRNAs. Elife 4: $\mathrm{e} 05005,2015$

19. Szklarczyk D, Gable AL, Lyon D, Junge A, Wyder S, Huerta-Cepas J, Simonovic M, Doncheva NT, Morris JH, Bork P, et al: STRING v11: Protein-protein association networks with increased coverage, supporting functional discovery in genome-wide experimental datasets. Nucleic Acids Res 47D: D607-D613, 2019. 
20. Shannon P, Markiel A, Ozier O, Baliga NS, Wang JT, Ramage D, Amin N, Schwikowski B and Ideker T: Cytoscape: A software environment for integrated models of biomolecular interaction networks. Genome Res 13: 2498-2504, 2003.

21. Lánczky A, Nagy Á, Bottai G, Munkácsy G, Szabó A, Santarpia L and Győrffy B: miRpower: A web-tool to validate survival-associated miRNAs utilizing expression data from 2178 breast cancer patients. Breast Cancer Res Treat 160: 439-446, 2016.

22. Rueden CT, Schindelin J, Hiner MC, DeZonia BE, Walter AE, ArenaET and Eliceiri KW: Image J2: Image J for the next generation of scientific image data. BMC Bioinformatics 18: 529, 2017.

23. Salam NK, Nuti R and Sherman W: Novel method for generating structure-based pharmacophores using energetic analysis. J Chem Inf Model 49: 2356-2368, 2009.

24. Irwin JJ, Sterling T, Mysinger MM, Bolstad ES and Coleman RG: ZINC: A free tool to discover chemistry for biology. J Chem Inf Model 52: 1757-1768, 2012.

25. Wang Y, Wang X, Xiong Y, Li CD, Xu Q, Shen L, Chandra Kaushik A and Wei DQ: An Integrated Pan-Cancer Analysis and Structure-Based Virtual Screening of GPR15. Int J Mol Sci 20: 6226, 2019.

26. Yu Y, Luo W, Yang ZJ, Chi JR, Li YR, Ding Y, Ge J, Wang X and Cao XC: miR-190 suppresses breast cancer metastasis by regulation of TGF- $\beta$-induced epithelial-mesenchymal transition. Mol Cancer 17: 70, 2018.

27. Wang H, Tan Z, Hu H, Liu H, Wu T, Zheng C, Wang X, Luo Z, Wang J, Liu S, et al: microRNA-21 promotes breast cancer proliferation and metastasis by targeting LZTFL1. BMC Cancer 19 $738,2019$.

28. Xia Y, Wang Y, Wang Q, Ghaffar M, Wang Y, Sheng W and Zhang F: Increased miR-203-3p and reduced miR-21-5p synergistically inhibit proliferation, migration, and invasion in esophageal cancer cells. Anticancer Drugs 30: 38-45, 2019.

29. Huang W, Wu Y, Cheng D and He Z: Mechanism of epithelial mesenchymal transition inhibited by miR 203 in non small cell lung cancer. Oncol Rep 43: 437-446, 2020.

30. Currie E, Schulze A, Zechner R, Walther TC and Farese RV Jr: Cellular fatty acid metabolism and cancer. Cell Metab 18 $153-161,2013$.
31. Chen TT and Li H: Fatty acid metabolism and prospects for targeted therapy of cancer. Eur J Lipid Sci Technol 119: 1600366, 2017.

32. Chen JC and Wu X: [miR-203 inhibits lung cancer cell metastasis by targeting fatty acid binding protein 4]. Nan Fang Yi Ke Da Xue Xue Bao 38: 578-583, 2018 (In Chinese).

33. Mikalayeva V, Ceslevičienè I, Sarapinienė I, Žvikas V, Skeberdis VA, Jakštas V and Bordel S: Fatty Acid Synthesis and Degradation Interplay to Regulate the Oxidative Stress in Cancer Cells. Int J Mol Sci 20: 1348, 2019.

34. Moore NL, Edwards DP and Weigel NL: Cyclin A2 and its associated kinase activity are required for optimal induction of progesterone receptor target genes in breast cancer cells. J Steroid Biochem Mol Biol 144B: 471-482, 2014.

35. Sun NK, Huang SL, Chang PY, Lu HP and Chao CC: Transcriptomic profiling of taxol-resistant ovarian cancer cells identifies FKBP5 and the androgen receptor as critical markers of chemotherapeutic response. Oncotarget 5: 11939-11956, 2014.

36. Hou $\mathbf{J}$ and Wang L: FKBP5 as a selection biomarker for gemcitabine and Akt inhibitors in treatment of pancreatic cancer. PLoS One 7: e36252, 2012.

37. Sato A, Asano T, Ito K and Asano T: 17-Allylamino-17-demethoxygeldanamycin and ritonavir inhibit renal cancer growth by inhibiting the expression of heat shock factor-1. Int J Oncol 41: 46-52, 2012.

38. Sato A, Asano T, Okubo K, Isono M and Asano T: Nelfinavir and Ritonavir Kill Bladder Cancer Cells Synergistically by Inducing Endoplasmic Reticulum Stress. Oncol Res 26: 323-332, 2018.

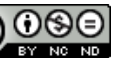

This work is licensed under a Creative Commons Attribution-NonCommercial-NoDerivatives 4.0 International (CC BY-NC-ND 4.0) License. 